\section{Enhancing biosurfactant production by hypersaline Bacillus amyloliquefaciens SK27 using response surface methodology and genetic algorithm}

\author{
Ruchira Malik and Savita Kerkar* \\ Department of Biotechnology, Goa University, Goa 403 206, India
}

The use of biosurfactants has been limited because of their low yield and high production cost. A central composite design was used to study the interactive effect of sucrose, yeast extract and sodium chloride which were the most influencing variables. Response surface analysis showed that the quadratic model with $R^{2}$ value of 0.9983 was fit for biosurfactant production. When genetic algorithm was used for maximization, the optimal activity (oil displacement zone) was found close to that obtained by response surface methodology, both of which were close to the predicted value. Biosurfactant production was enhanced by 1.2fold using these approaches.

Keywords: Bacillus amyloliquefaciens, biosurfactants, central composite design, genetic algorithm, response surface methodology.

BIOSURFACTANTS are surface-active amphiphilic molecules produced by various microorganisms. They are produced on the cell surface of the organism or excreted extracellularly, and have hydrophilic and hydrophobic moieties. Biosurfactants have the ability to accumulate between liquid phases and are capable of reducing surface and interfacial tension ${ }^{1}$. They are classified according to their chemical structure and microbial origin. In recent years, work is focused on their applications in various areas, such as oil recovery, food, cosmetics, agriculture, pharmaceutical industry, petro-chemistry and so on $^{2,3}$. Biosurfactants have numerous advantages compared to chemically synthesized surfactants, which include lower toxicity, biodegradability, being environmentfriendly, having better stability and tolerance to a wide range of $\mathrm{pH}$, temperature and salinity values ${ }^{4}$.

Despite numerous advantages and industrial applications, the utilization of biosurfactants has been restricted because of their low yield and high production cost ${ }^{5}$. One of the methods to overcome these limitations is to determine the appropriate nutrients at their optimal levels and ideal culture conditions for growth and production of biosurfactants ${ }^{6}$. Medium composition not only influences the growth and metabolism of the culture, but also increases the product yield. Thus, medium optimization is a standout

*For correspondence. (e-mail: drsavitakerkar@gmail.com) amongst the most crucially explored phenomena, that is completed before any large-scale metabolite production. For structuring a production medium, the most suitable fermentation conditions (e.g. $\mathrm{pH}$, temperature, agitation speed, etc.) and medium components (e.g. carbon, nitrogen, etc.) must be recognized and optimized accordingly ${ }^{7}$. This can be accomplished utilizing a wide range of strategies, from a classical 'one-factor-at-a-time' to present-day statistical and mathematical techniques, viz. genetic algorithm (GA), etc.

Optimization of culture conditions by the classical 'one-factor-at-a-time' (OFAT) method involves changing one factor while keeping all other factors constant. The advantage of this method is its simplicity. The outcomes can be analysed by means of simple graphs without using any statistical analysis. This method primarily helps in the selection of the most influential parameters affecting production significantly; however, it is incapable for studying the interactive effects of several nutritional parameters $^{8}$. This limitation can be overcome by the use of a statistical approach like response surface methodology (RSM).

RSM is an extensively applied statistical approach for designing experiments, assessing interactive effects of variables, and foreseeing the ideal conditions through least number of experiments ${ }^{9}$. It helps develop a numerical model portraying the compound or biochemical system considering the variables and their interactive influence simultaneously and in a few experiments ${ }^{10}$. It is also easy and efficient compared to methodologies because of decrease in the quantity of experimental runs, making it an excellent statistical tool ${ }^{11,12}$.

GA is a metaheuristic method inspired from Charles Darwin's theory of natural evolution to solve both constrained and unconstrained optimization problems. It develops solutions to given problems by mimicking the metaphor of the natural biological evolutionary process. Each GA works on an underlying population of potential solution to evaluate fitness and enhance a new population through the use of genetic operators. This method has a fitness value, a real number which will evaluate how good a solution is to the given problem ${ }^{13}$. Currently, utilization of GA to the quadratic equation of RSM is wellknown for the optimization of process parameters ${ }^{14-16}$. Thus this study is aimed at optimizing the fermentation medium for enhancing biosurfactant production by hypersaline Bacillus amyloliquefaciens SK 27 using statistical approaches.

The bacterial isolate, B. amyloliquefaciens SK27 (MF599413) used in the study was previously isolated from water sample of Ribandar saltpans of Goa, India $\left(15^{\circ} 30.166 \mathrm{~N}\right.$ and $\left.73^{\circ} 51.245 \mathrm{E}\right)$. The isolate was maintained on nutrient agar (HiMedia M001) prepared with sea water $^{17}$

The basic medium used for optimization of the biosurfactant was prepared according to Zhang et al. ${ }^{18}$. A 
loopful of the bacterial culture was inoculated in $50 \mathrm{ml}$ basic medium (glucose $20 \mathrm{~g} / 1$, peptone $4 \mathrm{~g} / \mathrm{l}$, potassium dihydrogen phosphate $5 \mathrm{~g} / \mathrm{l}$, potassium phosphate dibasic $5 \mathrm{~g} / \mathrm{l}$, calcium chloride $0.08 \mathrm{~g} / 1$, magnesium sulphate heptahydrate $0.25 \mathrm{~g} / 1$ and sodium chloride $4 \mathrm{~g} / \mathrm{l}$ ) and incubated on an orbital shaker $\left(30^{\circ} \mathrm{C}, 120 \mathrm{rpm}\right)$. After $24 \mathrm{~h}$, the culture supernatant was collected by centrifugation $\left(6000 \mathrm{rpm}, 15 \mathrm{~min}\right.$ and $\left.4^{\circ} \mathrm{C}\right)$, and used further to check the presence of the biosurfactant ${ }^{19}$. All the chemicals used were of analytical grade (Hi-Media, India).

The production of biosurfactant was determined by oil spreading method, as explained by Morikawa et al. ${ }^{20}$. In a petri plate containing $20 \mathrm{ml}$ of distilled water, $20 \mu \mathrm{l}$ of crude oil was added, which formed a layer on the water surface. To this, $10 \mu \mathrm{l}$ of culture supernatant was added. The petri plate was left undisturbed for $2-3 \mathrm{sec}$ at room temperature and observed for oil displacement (oil-free clearing zone). The diameter of this clearing zone is directly proportional to the biosurfactant concentration ${ }^{21}$. The size of this clearing zone indicates the surfactant activity, also denoted as oil displacement activity. Distilled water was used as negative control (without surfactant) and Triton X-100 (1 mg/ml) was maintained as the positive control.

In order to screen the most influential variables for the production of biosurfactant, one-factor-at-a-time method (OFAT) was carried out ${ }^{22}$. Various process parameters such as $\mathrm{pH}$ of the medium $(5-9)$, temperature $\left(10-50^{\circ} \mathrm{C}\right)$, carbon source (glucose/glycerol/fructose/sodium citrate/ mannitol/starch/mannose/lactose/paraffin/sucrose), nitrogen source (yeast extract/meat extract/peptone/urea/beef extract/tryptone/sodium nitrate/glycine/soya peptone/casein), concentration of carbon $(0-2 \%)$, concentration of nitrogen $(0-1.8 \%)$ and $\mathrm{NaCl}$ concentration $(0-4 \%)$ were analysed one at a time, keeping other factors constant.

Once the critical factors were identified, RSM approach was used to study the interaction among three influencing variables, i.e. carbon source: sucrose $(A)$, nitrogen source: yeast extract $(B)$ and sodium chloride (C) selected from OFAT for biosurfactant production using $B$. amyloliquefaciens SK27. The other media components were kept constant, with a variation in the three influential components ${ }^{23}$. Central composite design was utilized to get a quadratic model, comprising tryouts in addition to a star arrangement to gauge quadratic effects and central points to appraise the pure process variability, with biosurfactant production as response. The software package 'Design Expert 11.0' (Minneapolis, USA) was used to analyse the experimental design by central composite design ${ }^{24}$. As indicated by this design, 20 experiments were carried out in triplicate for estimating the experimental uncertainty variance. The response value in each trial was the average of triplicates measured in centimetres. Each factor in the design was considered at three different levels $(-1,0,+1)$. The levels of three independent variables used were: sucrose $(A)$, yeast extract $(B)$ and sodium chloride $(C)$, each at three coded levels (Table 1). The relationships and interrelationships of these variables were determined by fitting the seconddegree polynomial equation to data obtained from 20 experiments using mean values of triplicate of each experiment conducted thrice at different occasions. Response surface plots were generated using the Design-Expert software. The second-order model equation was determined by Fischer's test and the coefficient of determination, $R^{2}$ determined the fitness of the polynomial model equation.

GA is a strategy for solving both constrained and unconstrained optimization problems which is based on natural selection, the process that drives biological evolution. It is a simple cycle of four stages consisting of creation, selection, crossover, mutation. The process begins with the creation operator generating an initial population, once the initial population is generated; the selection operator selects the fittest candidates to breed and further alter the population by applying crossover and mutation operator to develop the new population. The entire procedure proceeds until an appropriate outcome is achieved ${ }^{7}$. Optimum conditions were selected after evaluation of GA to attain adjusted fermentation conditions in the specified range of input parameters. The GA toolbox of MATLAB 9.4 (MathWorks, Inc., Natick, MA, USA) was applied in the modelling studies ${ }^{25}$.

RSM consists of a pragmatic method for developing, advancing and optimizing processes influenced by variable responses. It defines the effect of independent variables, alone or in combination, on the process and generates a mathematical model ${ }^{26,27}$. Prior knowledge of the process and process variables is important for achieving a realistic

Table 1. Central composite design of the experimental design

\begin{tabular}{|c|c|c|c|c|}
\hline Run & $A$ & $B$ & $C$ & $\begin{array}{l}\text { Oil displacement } \\
\text { zone }(\mathrm{cm})\end{array}$ \\
\hline 1 & 0 & 0 & 0 & 8.8 \\
\hline 2 & -1 & 0 & 0 & 8.2 \\
\hline 3 & +1 & +1 & 0 & 8.2 \\
\hline 4 & 0 & +1 & +1 & 7.1 \\
\hline 5 & 0 & 0 & 0 & 8.8 \\
\hline 6 & 0 & 0 & +1 & 8.36 \\
\hline 7 & 0 & 0 & 0 & 8.8 \\
\hline 8 & -1 & 0 & 0 & 8.26 \\
\hline 9 & 0 & 0 & +1 & 7.36 \\
\hline 10 & 0 & 0 & -1 & 8.4 \\
\hline 11 & 0 & 0 & 0 & 8.8 \\
\hline 12 & +1 & +1 & 0 & 8.16 \\
\hline 13 & 0 & 0 & -1 & 7.16 \\
\hline 14 & -1 & +1 & 0 & 8.26 \\
\hline 15 & -1 & +1 & -1 & 7.4 \\
\hline 16 & +1 & 0 & +1 & 7.2 \\
\hline 17 & 0 & 0 & 0 & 8.8 \\
\hline 18 & +1 & -1 & 0 & 7.2 \\
\hline 19 & -1 & -1 & +1 & 7.2 \\
\hline 20 & 0 & 0 & 0 & 8.8 \\
\hline
\end{tabular}



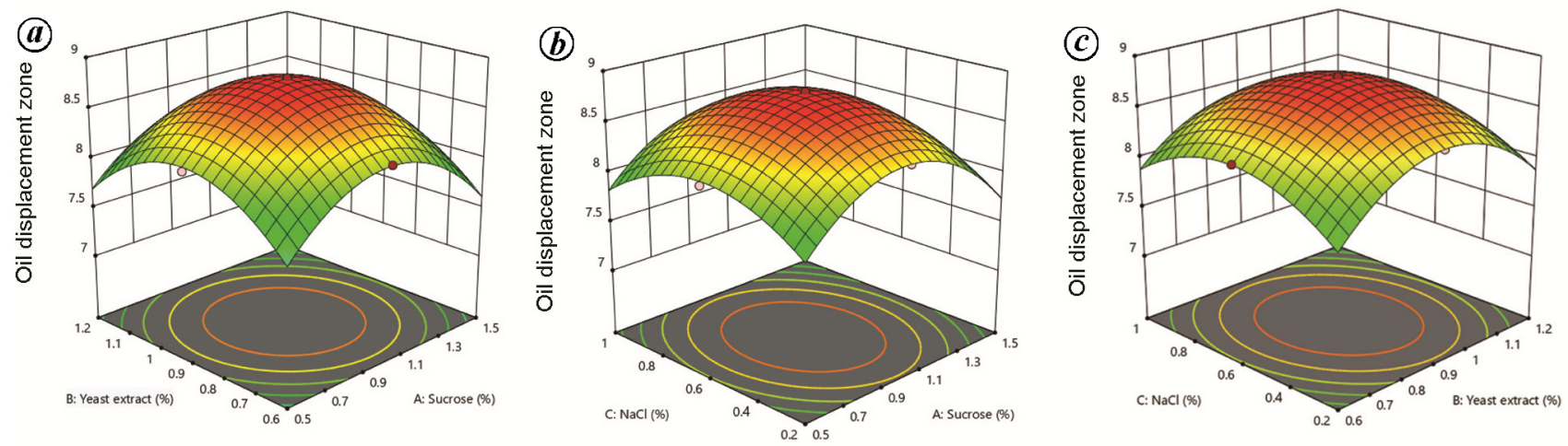

Figure 1. Surface plots of biosurfactant production (oil displacement zone). $\boldsymbol{a}-\boldsymbol{c}, 3 \mathrm{D}$ response surface curve plot of (a) sucrose and yeast extract; (b) sucrose and $\mathrm{NaCl}$; (c) yeast extract and $\mathrm{NaCl}$.

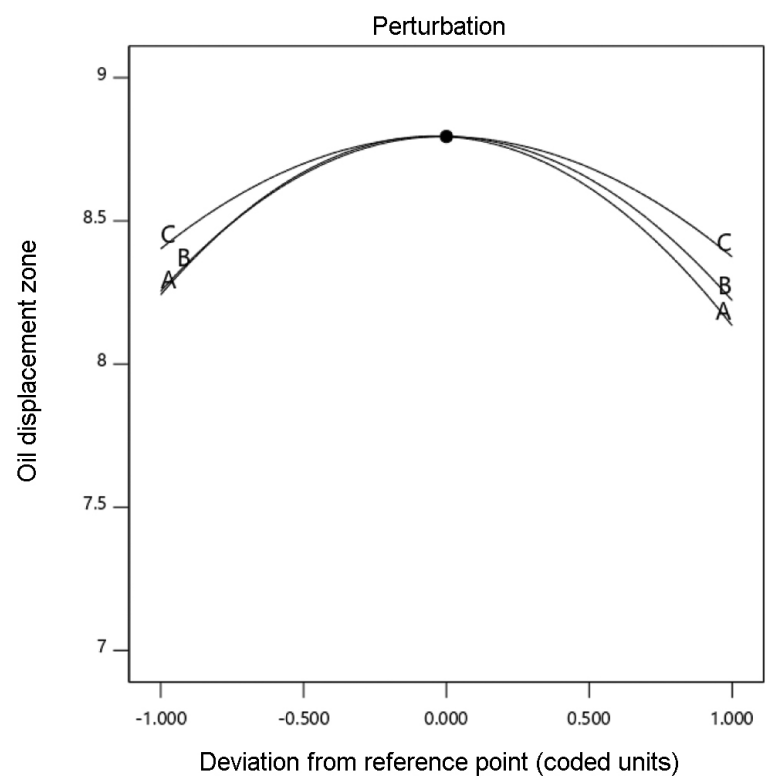

Figure 2. Pertubation graph showing the effect of each of the independent variables on biosurfactant production.

Table 2. Results of regression anaysis of experimental design

\begin{tabular}{lccr}
\hline Source & Mean square & $F$-value & Prob $>F$ \\
\hline Model & 1.01 & 661.63 & $<0.0001$ \\
$A$ - Sucrose & 0.0292 & 19.02 & 0.0014 \\
$B-$ Yeast extract & 0.0026 & 1.67 & 0.2254 \\
$C-\mathrm{NaCl}$ & 0.0020 & 1.28 & 0.2846 \\
$A B$ & 0.0040 & 2.64 & 0.1352 \\
$A C$ & 0.0000 & 0.0326 & 0.8603 \\
$B C$ & 0.0221 & 14.38 & 0.0035 \\
$A^{2}$ & 1.01 & 659.41 & $<0.0001$ \\
$B^{2}$ & 0.8512 & 555.14 & $<0.0001$ \\
$C^{2}$ & 0.4541 & 296.15 & $<0.0001$ \\
\hline
\end{tabular}

mode ${ }^{28}$. For this, the effect of different factors such as $\mathrm{pH}$, temperature, carbon and nitrogen sources, and concentration of carbon and nitrogen sources and sodium chloride was evaluated by OFAT. Sucrose and yeast extract had a significant effect on biosurfactant production compared to the other nine carbon and nitrogen sources. Factors such as $\mathrm{pH}$ and temperature did not have a considerable effect on biosurfactant production. Based on the identification of the effective variables, a central composite design was developed for the components affecting biosurfactant production, i.e. carbon source: sucrose $(A)$, nitrogen source: yeast extract $(B)$ and sodium chloride $(C)$.

Table 1 shows the experimental results of biosurfactant production by three-factor-two-level factorial experiment design with 6 replications of central point and 14 axial points.

RSM suggested a quadratic model to predict the responses by applying multiple regression analysis (Table 2).

$$
\begin{aligned}
Y= & +8.79-0.0540 * A-0.016^{*} B-0.014^{*} C \\
& -0.00225^{*} A^{*} B-0.0025^{*} A^{*} C-0.0525^{*} B^{*} C \\
& -0.6064^{*} A^{2}-0.5564 * B^{2}-0.4064^{*} C^{2}
\end{aligned}
$$

where $Y$ is the biosurfactant activity measured by oil clearing zone (cm); $A$ the sucrose concentration $(\%), B$ the yeast extract concentration (\%) and $C$ is the $\mathrm{NaCl}$ concentration (\%).

The ANOVA results revealed that the model is highly significant with an $F$-value of 661.63 and probability value of $<0.0001$ (Table 3). Further, the coefficient of determination $\left(R^{2}\right)$ was calculated to be 0.9983 , to examine the fitness of the model. The $R^{2}$ value was closer to 1.00 , which suggests that the model is stronger and better to predict the response ${ }^{29}$. The adjusted determination coefficient (adj $R^{2}=0.9968$ ) and predicted determination coefficient $\left(R^{2}=0.9680\right)$ were also satisfactory to confirm the significance of the model. Adjusted precision assesses the signal-to-noise ratio; a ratio of 62.501 shows satisfactory signal and therefore the model is significant for the process. A low value of 
Table 3. ANOVA for full quadratic model

\begin{tabular}{lccrrr}
\hline Source & Sum of squares & Mean square & DF & $F$-value & $P>F$ \\
\hline Model & 9.13 & 1.01 & 9 & 661.63 & $<0.0001$ \\
Residual & 0.0153 & 0.0015 & 10 & & \\
Lack of fit & 0.0153 & 0.0031 & 5 & \\
Pure error & 0.0000 & 0.0000 & 19 & \\
Total & 9.15 & & & \\
\hline
\end{tabular}

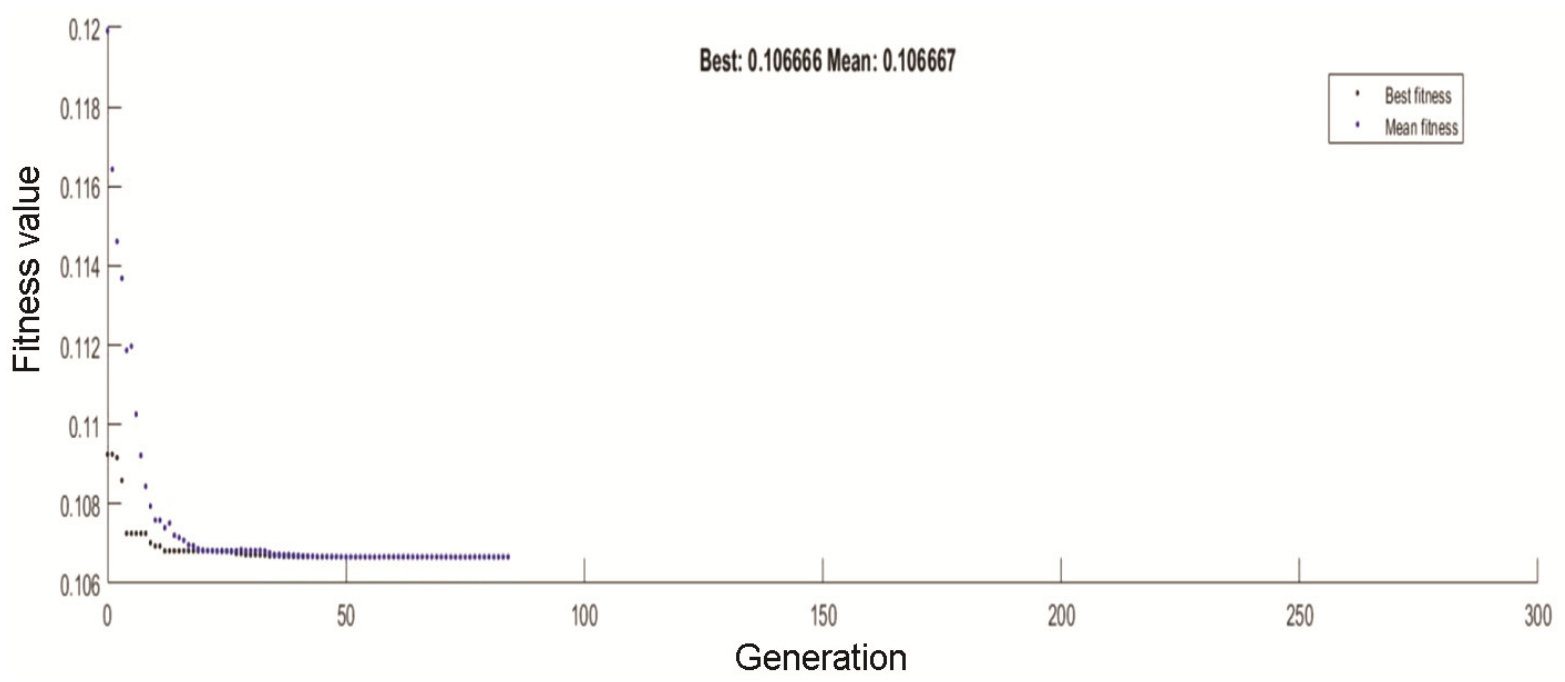

Figure 3. Fitness of the individual population.

coefficient of variation $(\mathrm{CV}=0.4889 \%)$ demonstrated that the experiment conducted was precise and reliable ${ }^{30}$.

Another method in RSM which gives a potential relationship between the variables is the three-dimensional surface plots. It displays the 3D relationship with predictor variables on the $x$ and $y$ scales, and the response $(z)$ variable.

The 3D response surface curve plots in Figure $1 a-c$ are a graphical representation of the regression equation. Each figure represents the effect of two variables, while the third is at zero level for maximum biosurfactant production. Figure $1 a$ shows the effect of sucrose and yeast extract on the biosurfactant production, keeping $\mathrm{NaCl}$ concentration constant at the middle level. Figure $1 b$ and $c$ also shows a similar trend. Perturbation graph shows the effect of each of the independent variables on biosurfactant production, while keeping the other variables at their respective middle point levels. The response surface plots and perturbation graph show that all the three variables (sucrose, yeast extract and $\mathrm{NaCl}$ ) play a significant role in the response (Figure 2).

Also, the perturbation graph shows the effect of each of the independent variables on biosurfactant production. Based on the response values, 3D plots and perturbation graph, it can be concluded that all the factors studied have a positive effect on biosurfactant production.
The optimal combination of the major media constituents for biosurfactant production as evaluated from the response surface and contour plots was sucrose $0.97 \%$, yeast extract $0.89 \%$ and $\mathrm{NaCl} 0.59 \%$. The model-predicted optimum activity was found to be $8.79 \mathrm{~cm}$ using the above-mentioned optimum concentration of the variables. Verification experiment was carried out using optimized conditions, which gave an activity of $8.8 \mathrm{~cm}$, close to the predicted value. A 1.2-fold increase in yield was observed, i.e. production was increased for 6.9 to $8.7 \mathrm{mg} / \mathrm{ml}$ after optimization with RSM, thereby proving the effectiveness of the model in media optimization.

GA is an optimization method which provides a fitness function to determine the optimum concentration of the media components. It is based upon the principle of 'survival of the fittest'. The RSM output data were further optimized using GA to obtain the best fermentation parameters. The nonlinear regression equation of oil displacement activity was optimized using GA optimization tool. Figure 3 represents fitness of the individual population. The oil displacement activity was found to be 8.76 at optimum process conditions which was close to that predicted by RSM. The application of RSM-GA hybrid statistical approaches thus resulted in a significant enhancement in biosurfactant production by $B$. amyloliquefaciens SK27. 


\section{RESEARCH COMMUNICATIONS}

Saibaba et al. ${ }^{31}$ applied hybrid RSM-GA-based technique for modelling and optimization of methylene blue dye removal. This method was found to be efficient and resulted in maximum dye removal of $96.9 \%$ (ref. 31). Shirodkar et al. $^{32}$ used RSM-GA hybrid method for enhancing the $\alpha$-amylase production by Thraustochytrium sp. AH-2. The study showed that there was 1.2-fold increase in $\alpha$-amylase production, which proved that these statistical experimental designs are efficient and valuable tools in optimizing medium composition ${ }^{32}$.

Several studies have been carried out on production of biosurfactants as they are environment-friendly compared to their synthetic counterparts. However on-field application is limited because of their low yield and high production cost. Thus, optimizing biosurfactant production is crucial for up-scaling the process. Optimization of the media components was carried out in the present study using RSM-GA amalgamated approach, which gave a 1.2-fold increase in the yield of biosurfactants. Thus, the statistical approach proved to be efficient, simple as well as time-saving for enhancing biosurfactant production from B. amyloliquefaciens SK27. To the best of our knowledge, there are no reports available on media optimization for maximizing the yield of biosurfactant from B. amyloliquefaciens using RSM-GA hybrid method, which is as a valuable tool for enhancing biosurfactant production.

1. Karnath, N. G. K., Deo, P. G. and Veenanadig, N. K., Microbial production of biosurfactant and their importance. Curr. Sci., 1999 77, 116-123.

2. Khopade, A., Ren, B., Liu, X. Y., Mahadik, K., Zhang, L. and Kokare, C., Production and characterization of biosurfactant from marine Streptomyces sp. B3. J. Colloid Interface Sci., 2012, 367, 311-318.

3. Satpute, S. K., Banpurkar, A. G., Dhakephalkar, P. K., Banat, I. M. and Chopade, B. A., Methods of investigating biosurfactants and bioemulsifiers: a review. Crit. Rev. Biotechnol., 2010, 30, 127-144; doi:10.3109/07388550903427280.

4. Mukherjee, S., Das, P. and Sen, R., Towards commercial production of microbial surfactants. Trends Biotechnol., 2006, 24(11), 509-515; doi:10.1016/j.tibtech.2006.09.005.

5. Mukherjee, S., Das, P., Sivapathasekaran, C. and. Sen, R., Enhanced production of biosurfactant by a marine bacterium on statistical screening of nutritional parameters. Biochem. Eng. J., 2008, 42, 254-260.

6. Kim, B. and Kim, J., Optimization of culture conditions for the production of biosurfactant by Bacillus subtilis JK-1 using response surface methodology. J. Kor. Soc. Appl. Biol. Chem., 2013, 56, 279-287; doi:10.1007/s13765-013-3044-6.

7. Singh, V., Haque, S., Niwas, R., Srivastava, A., Pasupuleti, M. and Tripathi, C. K. M., Strategies for fermentation medium optimization: an in depth review. Front. Microbiol., 2017, 7, 2087; doi:10.3389/fmicb.2016.02087.

8. Alikunju, A. P. et al., A statistical approach to optimize cold active $\beta$-Galactosidase production by an arctic sediment pscychrotrophic bacteria. Enterobacter ludwigii (MCC 3423) in cheese whey. Catal. Lett., 2018, 148(2), 712-724; doi:org/10.1007/ s10562-017-2257-4.

9. Myers, R. H. and Montgomery, D. C., Response Surface Methodology: Product and Process Optimization Using Designed Experiments, New York, John Wiley, USA, 2002, 2nd edn.
10. Vohra, A. and Satyanarayana, T., Statistical optimization of the medium components by response surface methodology to enhance phytase production by Pichia anomala. Process Biochem., 2002, 37, 999-1004.

11. Liyana-Pathirana, C. and Shahidi, F., Optimization of extraction of phenolic compounds from wheat using response surface methodology. Food Chem., 2005, 93, 47-56.

12. Montgomery, D., Design and Analysis of Experiments, John Wiley, New York, USA, 2005, 6th edn.

13. McCall, J., Genetic algorithms for modelling and optimisation. $J$. Comput. Appl. Math., 2005, 184(1), 205-222; doi:10.1016/j.cam. 2004.07.034.

14. Gaitonde, V. N., Karnik, S. R., Achyutha, B. T. and Siddeswarappa, B., GA application to RSM based models for burr size reduction in drilling. J. Sci. Indust. Res., 2005, 64, 347.

15. Franco-Lara, E., Link, H. and Weuster-Botz, D., Evaluation of artificial neural network for modelling and optimization of medium composition with a genetic algorithm. Proc. Biochem., 2006, 41, 2200-2206.

16. Moorthy, I. M. G. and Baskar, R., Statistical modeling and optimization of alkaline protease production from a newly isolated alkalophilic Bacillus species BGS using response surface methodology and genetic algorithm. Prep. Biochem. Biotechnol., 2013, 43, 293-314.

17. Fernandes, S., Kerkar, S., Leitao, J. and Mishra, A., Probiotic role of salt pan bacteria in enhancing the growth of Whiteleg Shrimp, Litopenaeus vannamei. Probiot. Antimicrob. Proteins, 2019, 1-15; doi.org/10.1007/s12602-018-9503-y.

18. Zhang, W., Zhang, X. and Cui, H., Isolation, fermentation optimization and performance studies of a novel biosurfactant producing strain Bacillus amyloliquefaciens, Chem. Biochem. Eng. Q., 2015, 29(3), 447-456.

19. Thavasi, R., Sharma, S. and Jayalakshmi, S., Evaluation of screening methods for the isolation of biosurfactant producing marine bacteria. J. Pet. Environ. Biotechnol., 2011, S1(001), 1-6; doi:10.4172/2157-7463.S1-001.

20. Morikawa, M., Daido, H., Takao, T., Murata, S., Shimonishi, Y. and Imanaka, T., A new lipopeptide biosurfactant produced by Arthrobacter sp. strain MIS 38. J. Bacteriol., 1993, 175, 64596466.

21. Youssef, N. H., Duncan, K. E., Nagle, D. P., Savage, K. N., Knapp, R. M. and McInerney, M. J., Comparison of methods to detect biosurfactant production by diverse microorganisms. $J$. Microbiol. Meth., 2004, 56, 339-347; doi:10.1016/j.mimet.2003. 11.001.

22. Irfan, M., Nadeem, M. and Syed, Q., One-factor-at-a-time (OFAT) optimization of xylanase production from Trichoderma virideIR05 in solid-state fermentation. J. Radiat. Res. Appl. Sci., 2014, 7, 317-326; doi:10.1016/j.jrras.2014.04.004.

23. Fernandes, M. S. and Kerkar, S., Enhancing the anti-tyrosinase activity of a hypersaline Kitasatospora sp. SBSK430 by optimizing the medium components. Curr. Sci., 2019, 116(4), 649-653; doi: $10.18520 / \mathrm{cs} / \mathrm{v} 116 / \mathrm{i} 4 / 649-653$.

24. Lee, N. K., Statistical optimization of medium and fermentation conditions of recombinant Pichia pastoris for the production of xylanase. Biotechnol. Bioprocess Eng., 2018, 23(1), 55-63; doi:10.1007/s12257-017-0262-5.

25. Srivastava, A. et al., Response surface methodology-genetic algorithm based medium optimization, purification, and characterization of cholesterol oxidase from Streptomyces rimosus. Sci. Rep., 2018, 8(1), 10913

26. Anjum, M. F., Tasadduq, I. and Al-Sultan, K., Response surface methodology: a neural network approach. Eur. J. Oper. Res., 1997, 101, 65-73.

27. Bas, D. and Boyac, I. S., Modeling and optimization I: usability of response surface methodology. J. Food Eng., 2007, 78, 836845 . 
28. Akhnazarova, S. and Kefarov, V., Experiment Optimization in Chemistry and Chemical Engineering, Mir Publishers, Moscow, Russia, 1982

29. Zhu, Z., Zhang, G., Luo, Y., Ran, W. and Shen, Q., Production of lipopeptides by Bacillus amyloliquefaciens XZ-173 in solid state fermentation using soybean flour and rice straw as the substrate. Bioresour. Technol., 2012, 112, 254-260; doi:10.1016/j.biortech. 2012.02.057.

30. Box, G. E. and Wilson, K., On the experimental attainment of optimum conditions. J. R. Stat. Soc. Series. B, 1951, 13, 1-45.

31. Saibaba, K. V. N. and King, P., Modelling and optimization of dye removal process using hybrid response surface methodology and genetic algorithm approach. J. Fundam. Renew. Energy Appl., 2014, 4(01), doi:10.4172/2090-4541.1000126.

32. Shirodkar, P. V. and Muraleedharan, U. D., Enhanced $\alpha$-amylase production by a marine protist, Ulkenia sp. using response surface methodology and genetic algorithm. Prep. Biochem. Biotechnol., 2017, 47(10), 1043-1049; doi:10.1080/10826068.

ACKNOWLEDGEMENT. We thank the Head, Department of Biotechnology, Goa University for providing funds and facilities.

Received 26 March 2019; revised accepted 20 June 2019

\section{South Asian subtropical low-level jet: influence on regional hydrology and aerosol optical depth}

\author{
T. R. Anoop ${ }^{1}$, C. K. Unnikrishnan ${ }^{1, *}$, \\ Karumuri Ashok ${ }^{2}$, K. K. Ramachandran ${ }^{1}$ and \\ T. N. Prakash ${ }^{1}$ \\ ${ }^{1}$ National Centre for Earth Science Studies, Akkulam, \\ Thiruvananthapuram 695011 , India \\ ${ }^{2}$ University of Hyderabad, Central University P.O., \\ Prof. C.R. Rao Road, Gachibowli, Hyderabad 500 046, India
}

A 38-year high-resolution wind climatology shows consistent occurrence of hitherto unreported South Asian subtropical low-level jets (SASLLJs) over the $31^{\circ}-35^{\circ} \mathrm{N}$ and $60^{\circ}-62^{\circ} \mathrm{E}$ latitude-longitude region, occurring throughout the year are the northerly $L L J$ (NLLJs) and southerly LLJs (SLLJs). The NLLJ is persistent mostly during the monsoon season. The NLLJ is frequent, unlike the SLLJ. The synoptic heat low in South Asia $\left(62^{\circ}-66^{\circ} \mathrm{E}\right.$ and $\left.27^{\circ}-32^{\circ} \mathrm{N}\right)$ and the anticyclone over Turkmenistan $\left(40^{\circ}-50^{\circ} \mathrm{N}\right.$ and $50^{\circ}-$ $60^{\circ} \mathrm{E}$ ) are critical in inducing the NLLJ. Regional topography permits direct mid-latitude air intrusion

*For correspondence. (e-mail: unnikrishnan.ck@ncess.gov.in) into the subtropical region. Importantly, we find that the NLU results in enhancing the transportation of dust to the Indian subcontinent and the northern part of the Arabian Sea due to wind blows. Long-term NLLJ intensity displays waning trend because of the weakening of pressure gradients between the heat low in South Asia and over Turkmenistan.

Keywords: Aerosol optical depth, low-level jet, regional hydrology, rainfall.

REGIONAL wind systems play a critical role in the climate and hydrology of an area ${ }^{1-3}$. The importance of low-level jets (LLJs) in impacting regional climate has been well recognized in many areas around the world ${ }^{4}$. The occurrence of regional wind patterns in Asia has an enormous consequence on oil exploration, agriculture and the economy of a region $^{5-7}$. The monsoon LLJ and Shamal wind over the Asian region have been well documented ${ }^{3,5,8}$. Previous studies suggest the existence of low-level northerly winds from June to September over Southwest Asia in the Iran-Afghanistan border area ${ }^{9,10}$. The northerly wind over Iran region is known as the Sistan wind or the "wind of 120 days" ${ }^{10}$. A recent study has examined the influence of Asian winds on the ecosystem in South Asia ${ }^{11}$. It also discusses about a Levar wind near the IranAfghanistan region reaching up to the northern part of the Arabian Sea. However, these studies are based on a dataset for a shorter period during the monsoon season. The analyses of 38-year multiple atmospheric reanalysis data showed two LLJ spells in the region, and we report them here as South Asian Subtropical LLJ (SASLLJ). The SASLLJ spells can be categorized into two types based on the direction as northerly and southerly (NLLJ and SLLJ respectively).

South Asia has relatively high population density, where freshwater availability mainly depends on the precipitation over the region. It has been recognized that even a small rainfall anomaly can influence the economy of South Asia ${ }^{12,13}$. Many regional winds influence the precipitation pattern and hydrology of the region ${ }^{1,14,15}$. On a different note, a study showed that aerosols from the Sahel region influence the Indian summer monsoon climate $^{16}$. In the present study, potential influence of the aforementioned wind patterns in West Asia, and its synoptic pattern on rainfall anomalies and aerosol transport into South Asia are examined. The study also analyses aerosol optical depth (AOD) as observed by the MODIS satellite ${ }^{17}$, which realized in understanding composite anomalies over South Asia.

Here we analyse the long-period characteristics of SASLLJs near the Iran-Afghanistan region using multiple reanalysis data. The role of SASLLJ synoptic conditions on regional hydrology and AOD over Asia is also examined. The long-term trend of SASLLJ intensity and its causes are presented. The European Centre for Medium-Range Weather Forecasts (ECMWF), the ERA 